\title{
Formation of light (anti)nuclei
}

\section{J. Tjemsland ${ }^{a, *}$}

${ }^{a}$ Institutt for fysikk, NTNU,

Trondheim, Norway

E-mail: jonas.tjemsland@ntnu.no

The production mechanism of light nuclei, such as deuteron, helium-3, tritium and their antiparticles, has recently attracted an increased attention from the astroparticle and heavy ion communities. The expected low astrophysical background of light antinulei makes them ideal probes for exotic astrophysical processes, such as dark matter annihilations. At the same time, they can be used to measure two-nucleon correlations and density fluctuations in heavy ion collisions, which may shed light on the QCD phase diagram. Motivated by the importance of light antinuclei in cosmic ray studies, we developed a new coalescence model for light (anti)nuclei that includes both the size of the formation region, which is process dependent, and momentum correlations in a semiclassical picture. We have employed the model as an afterburner to the event generators Pythia 8 and QGSJET II, and find that the model agrees well with experimental data on antideuteron and antihelium-3 production in $e^{+} e^{-}, p p, p \mathrm{Be}$ and $p \mathrm{Al}$ collisions at various energies. In this paper, we review this model and update existing fits to experimental data based on new insights.

Tools for High Energy Physics and Cosmology - TOOLS2020

2-6 November, 2020

Institut de Physique des 2 Infinis (IP2I), Lyon, France

${ }^{*}$ Speaker 


\section{Introduction}

The production mechanism of light nuclei, such as deuteron, helium-3, tritium and their antiparticles, has attracted attention from both the astroparticle and heavy ion communities. These particles are of particular importance in cosmic ray studies due to their low expected background at small kinetic energies, for a recent review see Ref. [1]. This makes them a promising detection channel for exotic physics, such as dark matter annihilations and decays. Currently, the measurement of astrophysical antinuclei is performed by the AMS-02 experiment on-board the International Space Station, while the balloon-borne GAPS experiment is expected to be launched in December $2022[2,3]$. Unfortunately, the AMS-02 collaboration has not yet published any results regarding antinuclei measurements, even though the expected antideuteron flux from secondary production and optimistic dark matter models is close to its estimated sensitivity [1]. In heavy ion collisions, (anti)nuclei measurements are of particular interest due to their small binding energies. This make them sensitive probes for two-nucleon correlations and density fluctuations that may shed light on the QCD phase diagram [4]. In order to correctly interpret the results of both collider and cosmic ray experiments, a precise description of the production mechanism is important. The focus in this work is on small interacting systems.

In Ref. [5], we developed a new coalescence model for deuteron, tritium and helium-3 based on the Wigner function representation of the produced nuclei states (the abbreviation WiFunC, short for Wigner Function with Correlations, will be used for this model). The model was later refined and applied in Ref. [6] for astrophysical processes, and in Ref. [7] for recent collider data. In contrast to existing production models, the WiFunC model includes both the size of the nucleon formation region and two-particle correlations in a semi-classical picture. In this work, the WiFunC model is reviewed.

This paper is structured as follows. In section 2 the standard coalescence model in momentum space commonly used for small interacting systems is discussed. This serves as a motivation for the WiFunC model, which is discussed in section 3. In subsection 3.4 we update and extend the fits to experimental data from Refs. [5, 6] based on new insights. The numerical implementation of the WiFunC model is outlined in section 4. Finally, we summarise in section 5.

\section{The coalescence model in momentum space}

The production of light nuclei ${ }^{1}$ is often described using coalescence models. In this type of models, a nucleus is produced from nucleons that have (nearly) completed their formation. Traditionally, the yield of a nucleus consisting of $Z$ protons and $N$ neutrons is parametrised as

$$
E_{A} \frac{\mathrm{d}^{3} N_{A}}{\mathrm{~d} P_{A}^{3}}=\left.B_{A}\left(E_{p} \frac{\mathrm{d}^{3} N_{p}}{\mathrm{~d} p_{p}^{3}}\right)^{Z}\left(E_{n} \frac{\mathrm{d}^{3} N_{n}}{\mathrm{~d} p_{n}^{3}}\right)^{N}\right|_{P_{A} / A=p_{n}=p_{p}}
$$

where $B_{A}$ is the so-called coalescence factor and $A=Z+N$ is the nucleon number. Motivated by the small binding energies of light nuclei, the coalescence condition is often applied in momentum

\footnotetext{
${ }^{1}$ Most of the discussions related to coalescence apply equally well to particles as to antiparticles, and the prescription "anti" will thus often be neglected.
} 
space for small interacting systems. In this case, two nucleons coalesce if their invariant momentum difference is less than the phenomenological coalescence momentum $p_{0}$ [8-10]. In the limit of isotropic nucleon yields, one can show that $B_{A} \propto p_{0}^{3(A-1)}$. This can in turn be improved by considering the coalescence condition per-event using momentum correlations provided by a Monte Carlo generator, as first proposed in Refs. [11, 12]. Furthermore, it was later noted in Ref. [13] that particles have to be close to the primary interaction to be able to coalesce, which essentially mean that weak decays should be considered as a separate cluster [14]. For heavy ion-collisions, an alternative scheme was developed where the coalescence factor instead scales with the nucleon emission volume as $B_{A} \propto V^{A-1}[15,16]$.

Clearly, the coalescence model in momentum space is purely phenomenological and classical. Furthermore, the coalescence momentum $p_{0}$ should be independent of both the process type and the center of mass (c.m.) energy to be predictive, which is not the case. In Fig. 1 we plot the coalescence momentum $p_{0}$ obtained by a fit to various experimental data in Refs. [6, 17]. As noted in Ref. [17], the result in Fig. 1 may suggest that the coalescence momentum $p_{0}$ exhibits an energy dependence: $p_{0}$ rapidly decreases to zero for small $\sqrt{s}$. There are, however, two problems with this conclusion: the process dependence has not been accounted for and the energy dependence has not been explained. These are two motivations for the WiFunC model, in which the apparent energy dependence is removed by taking into account the process dependence. ${ }^{2}$ More importantly, the WiFunC model is semi-classical and has a clear microphysical interpretation that can be used to predict its free parameter.

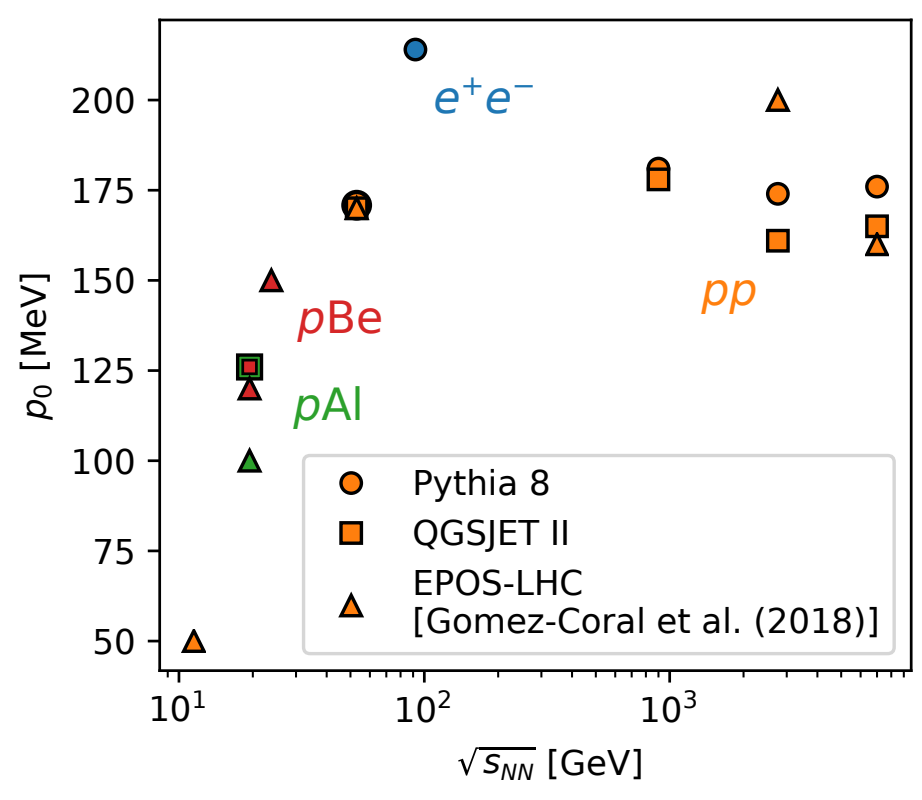

Figure 1: Best fit value of $p_{0}$ for experiments on $p p$ (orange), $e^{+} e^{-}$(blue), $p N$ (red and green) interactions using QGSJET II (squares), Pythia 8.2 (circles) and EPOS LHC (triangles) from Refs. [6, 17].

\footnotetext{
${ }^{2}$ Note that we have not considered the data point at $\sim 12 \mathrm{GeV}$ from Ref. [18]. An independent confirmation of this data point will thus falsify our model.
} 


\section{The WiFunC model}

\subsection{Motivation}

One of the main goals of the WiFunC model is to take into account both two-particle correlations and the size of the nucleon formation region when describing the production of light nuclei. Two-particle correlations are naturally important for small interacting systems as the production is highly non-isotropic. Meanwhile, the importance of the formation region becomes apparent when inspecting the relevant timescales of the problem [19]: the initial hard scattering occurs on timescales $t_{\mathrm{ann}} \sim 1 / \sqrt{s}$, and is succeeded by a perturbative cascade with a characteristic momentum transfer $\Lambda_{\mathrm{QCD}}^{2} \ll\left|q^{2}\right| \ll s$. This means that the largest time and distance scales in the problem is related to the hadronisation length, $L_{\text {had }} \sim \gamma L_{0}$ with $L_{0} \sim 1 \mathrm{fm}$. The light nuclei is in turn in the coalescence picture formed by nucleons that have (nearly) completed their formation. Since the nucleus wave functions have a size $r_{\text {rms }}^{N} \sim$ few fm comparable to the hadronisation length, the emission volume should not be neglected even in point-like processes.

\subsection{The underlying physics and theory}

The WiFunC model is based on the quantum mechanical description of coalescence, as reviewed in e.g. Ref. [20]. The deuteron ${ }^{3}$ spectrum can in this picture be found by projecting the reduced density matrix describing the nucleons onto the nucleus density matrix: $\mathrm{d}^{3} N_{d} / \mathrm{d} P_{d}{ }^{3}=\operatorname{tr}\left(\rho_{d} \rho_{\text {nucl }}\right)$. Rewriting this in terms of the two-nucleon Wigner function, $W_{n p}$, and the deuteron Wigner function, $\mathcal{D}$, leads to

$$
\frac{\mathrm{d}^{3} N_{d}}{\mathrm{~d} P_{d}^{3}}=\frac{3}{8} \int \mathrm{d}^{3} r_{d} \int \frac{\mathrm{d}^{3} q \mathrm{~d}^{3} r}{(2 \pi)^{6}} \mathcal{D}(\mathbf{r}, \mathbf{q}) W_{n p}\left(\mathbf{P}_{d} / 2+q, \mathbf{P}_{d} / 2-\mathbf{q}, \mathbf{r}_{n}, \mathbf{r}_{p}\right),
$$

where the factor $3 / 8$ is obtained by averaging over spin and isospin. It is convenient to approximate the deuteron wave function as a Gaussian, in which case $\mathcal{D}(\mathbf{r}, \mathbf{q})=8 \exp \left\{-r^{2} / d^{2}-q^{2} d^{2}\right\}$ with $d \simeq 3.2 \mathrm{fm}$. The effect of two-particle correlations on the coalescence probability is, however, sensitive to the shape of the deuteron wave function. Thus, a more accurate wave function should be used when momentum correlations are taken into account, using e.g. the sum of two Gaussians as introduced in Ref. [5]. ${ }^{4}$

In order to proceed, one has to provide a prescription for the nucleon Wigner function. In the WiFunC model, a factorisation of the momentum and position dependence in the Wigner function is assumed, ${ }^{5}$

$$
W_{n p}=H_{n p}\left(\mathbf{r}_{n}, \mathbf{r}_{p}\right) G_{n p}\left(\mathbf{P}_{d} / 2+\mathbf{q}, \mathbf{P}_{d} / 2-\mathbf{q}\right),
$$

and the spatial nucleon distributions are assumed to be uncorrelated, $H_{n p}\left(\mathbf{r}_{n}, \mathbf{r}_{p}\right)=h\left(\mathbf{r}_{n}\right) h\left(\mathbf{r}_{p}\right)$. In turn, the spatial distributions are taken to be Gaussians,

$$
h(\mathbf{r})=\left(2 \pi \sigma^{2}\right)^{-3 / 2} \exp \left\{-\frac{r^{2}}{2 \sigma^{2}}\right\} .
$$

\footnotetext{
${ }^{3}$ For concreteness, only the production of deuteron is discussed in this subsection. The model for helium- 3 and tritium is derived using the same procedure.

${ }^{4}$ The numerical implementation discussed in section 4 includes such an improved wave function.

${ }^{5}$ Note that this implies a transition to a semi-classical treatment.
} 
Inserting the ansätze (3) and (4) into Eq. (2) leads to the WiFunC model,

$$
\frac{\mathrm{d}^{3} N_{d}}{\mathrm{~d} P_{d}^{3}}=\frac{3 \zeta}{(2 \pi)^{6}} \int \mathrm{d}^{3} q e^{-q^{2} d^{2}} G_{n p}\left(\mathbf{P}_{d} / 2+\mathbf{q}, \mathbf{P}_{d} / 2-\mathbf{q}\right) .
$$

The function $\zeta$ describes the distribution of the nucleons, and it is in general given by

$$
\zeta\left(\sigma_{\|}, \sigma_{\perp}, d\right)=\sqrt{\frac{d^{2}}{d^{2}+4 \sigma_{\perp}^{2} /\left(\cos ^{2} \theta+\gamma^{2} \sin ^{2} \theta\right)}} \sqrt{\frac{d^{2}}{d^{2}+4 \sigma_{\perp}^{2}}} \sqrt{\frac{d^{2}}{d^{2}+4 \sigma_{\|}^{2}}},
$$

where $\theta$ is the angle between the deuteron momentum and the $z$-axis of the c.m. frame of the particle collision, while $\sigma_{\perp / \|}$ describes the characteristic spatial spread of nucleons in the perpendicular/parallel direction in the lab frame. ${ }^{6}$ The effective Lorentz boost of the transverse spread is included to account for the boost between the lab frame and the deuteron rest frame [5].

In Eq. (5) one particular choice for the semi-classical emission volume was used. Some event generators like Pythia 8 [21, 22] and UrQMD [23] have implemented a semi-classical treatment of the particle trajectories in the event. In this case, one can instead evaluate directly

$$
\frac{\mathrm{d}^{3} N_{d}}{\mathrm{~d} P_{d}^{3}}=3 \int \frac{\mathrm{d}^{3} q \mathrm{~d}^{3} r}{(2 \pi)^{6}} \mathrm{e}^{-r^{2} / d^{2}-q^{2} d^{2}} W_{n p}\left(\mathbf{P}_{d} / 2+q, \mathbf{P}_{d} / 2-\mathbf{q}, \mathbf{r}_{n}, \mathbf{r}_{p}\right),
$$

thereby relying on the spatial correlations provided by the event generator instead of Eqs. (3) and (4).

\subsection{Process dependence}

The free parameters in the WiFunC model, $\sigma_{\perp}$ and $\sigma_{\|}$, have the physical interpretation as the size of the formation region of nucleons. They will, in general, have a contribution from the perturbative cascade and hadronisation, and a contribution related to the finite size of the colliding particles:

$$
\sigma_{\|, \perp}^{2}=\sigma_{\|, \perp\left(e^{+} e^{-}\right)}^{2}+\sigma_{\|, \perp(\text { geom })}^{2}
$$

Here, the subscripts $\left(e^{+} e^{-}\right)$and (geom) denote respectively the point-like contribution from the cascade and the geometrical contribution from the finite size of the particles.

For point-like processes the spatial spread in the longitudinal direction is given by $\sigma_{\|} \simeq R_{p} \simeq$ $1 \mathrm{fm}$. Meanwhile, the spread in the transverse direction comes from the random walk behaviour of the perturbative cascade. This means that $\sigma_{\perp} \simeq \Lambda_{\mathrm{QCD}}^{-1} \simeq 1 \mathrm{fm}$.

The geometrical contributions can be approximated by $[5,6]$

$$
\begin{aligned}
\sigma_{\perp \text { (geom) }}^{2} & \simeq \frac{2 R_{1}^{2} R_{2}^{2}}{R_{1}^{2}+R_{2}^{2}}, \\
\sigma_{\| \text {(geom) }}^{2} & \simeq \max \left\{R_{1}, R_{2}\right\},
\end{aligned}
$$

where $R_{1}$ and $R_{2}$ are the Gaussian radii of the parton clouds in the colliding particles. For nuclei, these can be well approximated as

$$
R_{A} \simeq a_{0} A^{1 / 3}
$$

${ }^{6}$ For annihilation processes, $\theta, \sigma_{\perp}$ and $\sigma_{\|}$is defined relative to the initial quark pair in the hard process. 
with $a_{0} \simeq 1 \mathrm{fm}$, where $A$ is the number of nucleons in the nucleus that take part in the interaction [24]. Thus, we can describe the coalescence of light nuclei using a single universal parameter by setting ${ }^{7}$

$$
\sigma \equiv \sigma_{\left(e^{+} e^{-}\right)}=a_{0}=\sigma_{(p p)} / \sqrt{2} .
$$

\subsection{Comparison with experimental data}

In Fig. 2 we plot the best fit of $\sigma$ to the experimental data considered in Refs. [5-7] as a function of the c.m. energy of the collision using Pythia 8 [21, 25] and QGSJET II [26, 27]. ${ }^{8}$ The data points are well described by a constant spread $\sigma=(1.0 \pm 0.1) \mathrm{fm}$, as expected by the WiFunC model. Thus, the apparent energy dependence seen in Fig. 1 is alleviated by taking into account the nucleon emission volume.

The WiFunC model improves significantly the fit to experimental data at large transverse momenta compared to the standard coalescence model. This is readily seen in Fig. 3, where the best fit to the invariant differential yield of antideuterons measured by the ALICE collaboration [28] is plotted using Pythia 8 and QGSJET II. Furthermore, it describes the behaviour of the nontrivial baryon emission volume as measured by the ALICE collaboration [7, 29]. This is particularly important as it provides a method of determining the parameter $\sigma$ independent of an event generator (orange triangle in Fig. 2).

\subsection{Comment on helium-3 and tritium production}

In contrast to the deuteron wave function, the helium-3 and tritium wave functions are not well known. Therefore, the rms charge radii $r_{\text {rms }}^{3} \mathrm{He}=1.96 \mathrm{fm}$ and $r_{\text {rms }}^{t}=1.76 \mathrm{fm}$ were used in Refs. $[5,6]$ to describe the wave functions as a Gaussian. ${ }^{9}$ This will lead to an artificially small spread $\sigma$ if in reality either the matter radius is smaller than the charge radius or the wave function is more peaked than a Gaussian.

In the coalescence picture, deuteron, helium-3 and tritium are expected to be created by nucleons with the same spread, and the parameter $\sigma$ should thus be the same for all three particles. In the previous subsection, we saw that the spread is already well determined by antideuteron experiments, which has been confirmed independently by the measurements on the baryon emission volume by the ALICE collaboration [7, 29]. Thus, we can allow ourselves to degrade $r_{\mathrm{rms}}^{3} \mathrm{He}$ to a free parameter to better describe the effective ground state of the nucleus. In the spirit of simplicity and for consistency with Eq. (11), we set $r_{\text {rms }}^{3}=b=3^{1 / 3} \sigma$. Remarkably, this choice leads to $\sigma=(0.93 \pm 0.04) \mathrm{fm}$ for Pythia 8 and $\sigma=(1.07 \pm 0.03)$ fm for QGSJET II when fitted to the antihelium-3 spectrum at $7 \mathrm{TeV}$ measured by the ALICE collaboration [28], consistent with the antideuteron fits in Fig. 2. These

\footnotetext{
${ }^{7}$ Notice the ambiguity of this choice: we expect in general $\sigma_{\|} \gtrsim \sigma_{\perp}$ and we could therefore have chosen a different scaling between the parameters. Nevertheless, we find that this choice well describes the physics and experimental data. If more accurate data and event generators become available in the future, a separate fit of $\sigma_{\perp}$ and $\sigma_{\|}$, or even the geometrical and point-like spread, may be needed.

${ }^{8}$ In addition to including also the data on helium production for QGSJET, we have made the following changes compared to Refs. [5, 6]. First, we now vary the spread $\sigma$ in $p N$ collisions on an event by event basis depending on the number of nucleons that participate in the interaction using Eq. (11). Second, we now use the distribution factor $\zeta$ with boost in only one transverse component [Eq. (6)]. Finally, the size of the helium wave function is treated as discussed in section 3.5 .

${ }^{9}$ Since the spectra of tritium and helium-3 are expected to be similar, it is convenient to assume $r_{\mathrm{rms}}^{t}=r_{\mathrm{rms}}^{3} \mathrm{He}$ [5].
} 


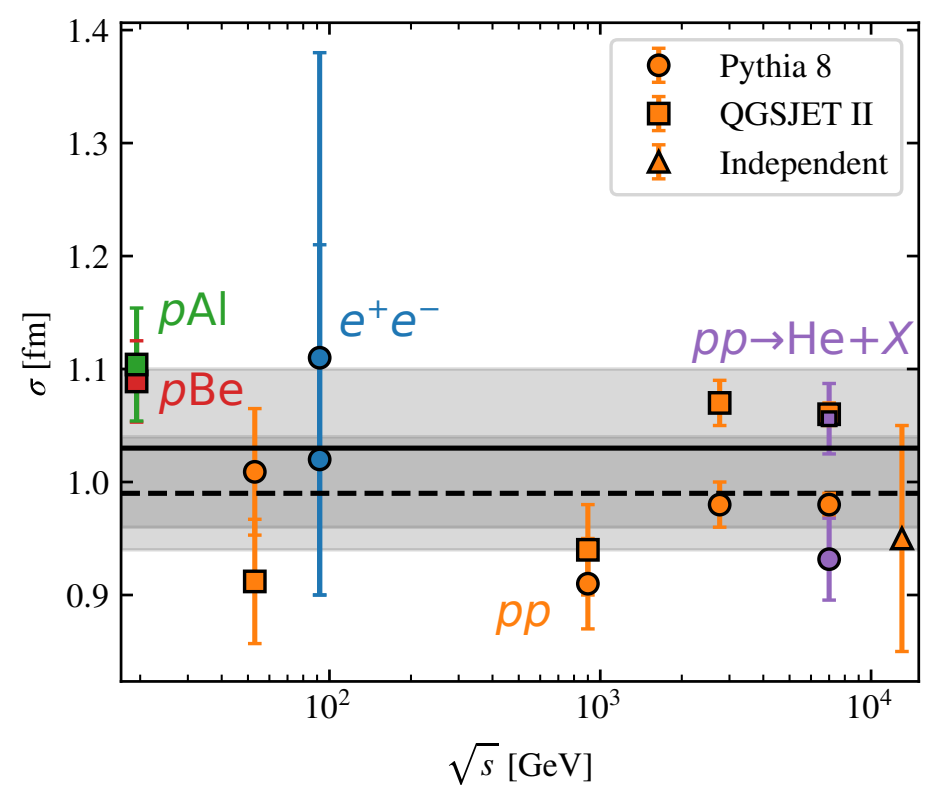

Figure 2: The best fit of $\sigma$ to experimental data on antinuclei production in $e^{+} e^{-}$(blue), $p p$ (orange), $p \mathrm{Be}$ (red) and $p \mathrm{Al}$ (green) collisions using Pythia 8 (circles) and QGSJET II (squares). The result obtained in Ref. [7] from comparison with the measured baryon emission volume is also shown (triangle). The mean of the data points for Pythia (dashed line) and QGSJET (solid line) and their standard deviations (transparent gray regions) are plotted without regards to the uncertainties in order to visualise the variation.

values corresponds to a rms radius $1.3-1.6 \mathrm{fm}$, which is by no means far-fetched. ${ }^{10}$ Nevertheless, it should be emphasised that theoretical uncertainties related to the wave function has not been properly accounted for.

\subsection{Comment on thermal models}

The production of light nuclei in heavy ion collisions is often described using thermal statistical models, where it is assumed that the nuclei are produced around chemical freeze-out in an expanding "fireball" consisting of a quark gluon plasma. Such models are motivated by the observation that the nucleus spectra are near thermal with a similar freeze-out temperature as for nucleons and mesons [30]. It is, however, hard to resolve the issue on how particles with small binding energies can survive the freeze-out process. Furthermore, since the hadron spectra already are thermallike, coalescence models also predicts thermal-like nucleus spectra up to a quantum mechanical correction factor [15].

Intriguingly, recent observations show characteristic signs for the production of a quark gluon plasma in small interacting systems, such as $p p$ and $p \mathrm{~Pb}$ collisions [31]. Such hints have been used as a motivation for using thermal models to describe the nucleus production even in small interacting systems [1, 32-34]. However, many of these, e.g. the behaviour of the $B_{2}$ factor as a function of multiplicity and transverse momentum in $p p$ collisions [34] and the decrease in the

\footnotetext{
${ }^{10}$ This change may lead to an increase in the cosmic ray antihelium spectra in Ref. [6] by a factor of a few.
} 

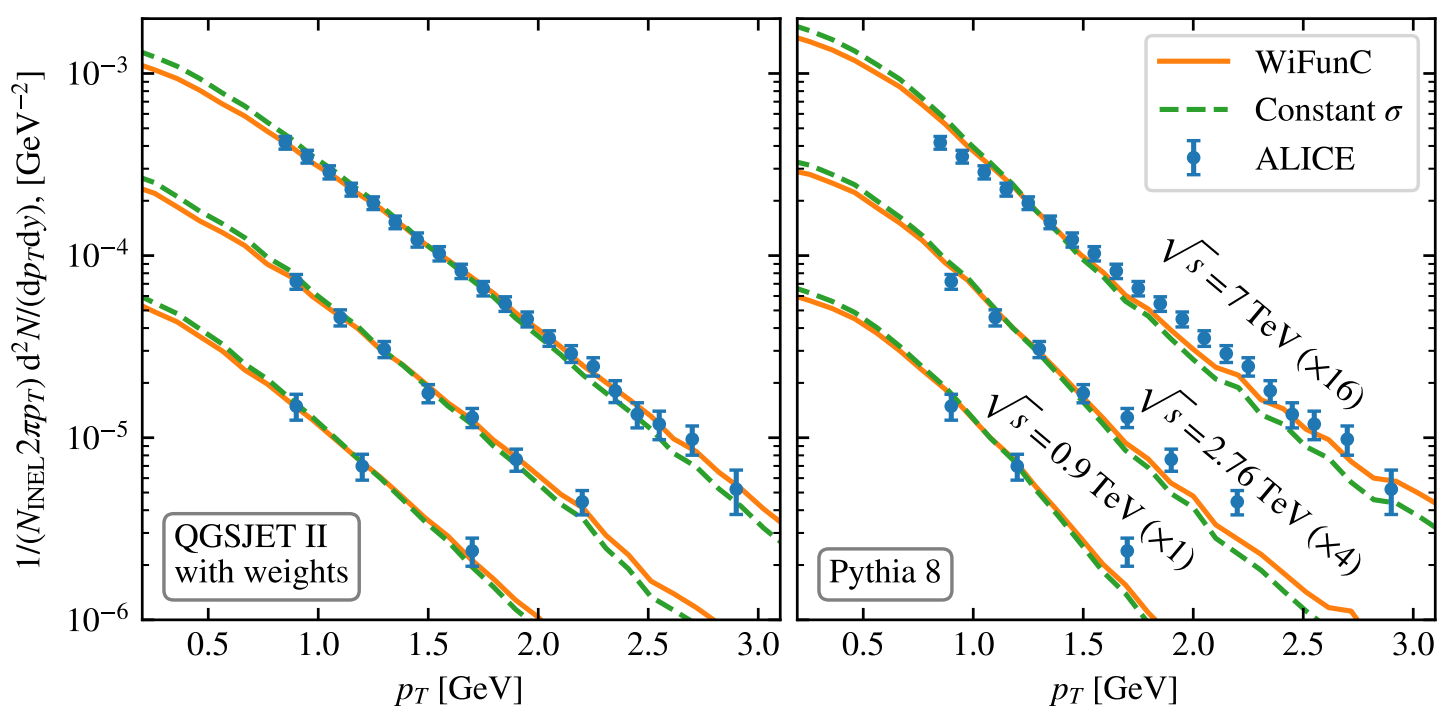

Figure 3: The best fit of the WiFunC model using QGSJET II (left) and Pythia 8.2 (right) to the invariant differential yield of antideuterons as a function of the transverse momentum measured by the ALICE collaboration [28] at $0.9,2.76$ and $7 \mathrm{TeV}$ (blue points). The data are multiplied by a constant factor to make the figure clearer. In the fit using QGSJET, a weight is included in order to better reproduce corresponding nucleon measurements [6]. The $\mathrm{WiFunC}$ model (orange line) reproduce well the data points. The WiFunC model without any Lorentz boost gives a similar fit as the standard coalescence model and is shown for comparison (green dashed line).

baryon emission volume with increasing transverse momentum [29], is also naturally described by the WiFunC model [7]. Moreover, the hints are irrelevant for astrophysical processes since they are at present only observed at high energies and multiplicities.

Regardless of the underlying hadron production, the WiFunC model is applicable as long as the nuclei are produced in a coalescence process. It is thus worth pointing out that it is argued in Ref. [35] that two-particle correlation experiments can be used to validate the coalescence hypothesis, and that the success of the femtoscopy analysis is strong evidence that coalescence is a major production mechanism for nuclei.

\section{Numerical implementation of the WiFunC model}

In this section, we briefly summarise how the WiFunC model can be evaluated for deuteron, helium-3 and tritium. The momentum of the nucleons produced in a particle collision is to be computed on an event-by-event basis using an event generator. Double counting can in all relevant applications be neglected [5].

In the WiFunC model, the probability that a given proton-neutron pair coalesce is

$$
w=3 \Delta \zeta_{1} \mathrm{e}^{-d_{1}^{2} q^{2}}+3(1-\Delta) \zeta_{2} \mathrm{e}^{-d_{2}^{2} q^{2}},
$$

where

$$
\zeta_{i}=\sqrt{\frac{d_{i}^{2}}{d_{i}^{2}+4 \tilde{\sigma}_{\perp}^{2}}} \sqrt{\frac{d_{i}^{2}}{d_{i}^{2}+4 \sigma_{\perp}^{2}}} \sqrt{\frac{d_{i}^{2}}{d_{i}^{2}+4 \sigma_{\|}^{2}}}
$$


and $\tilde{\sigma}_{\perp}^{2}=\sigma_{\perp}^{2} /\left(\cos ^{2} \theta+\gamma^{2} \sin ^{2} \theta\right)$. The quantities that vary event-by-event are indicated in Fig. 4: $|\mathbf{q}|$ is the momentum of the nucleons in the deuteron rest frame, $\theta$ is the angle between the deuteron momentum and the $z$-axis of the particle collision in the c.m. frame of the collision and $\gamma$ is the deuteron Lorentz factor in the c.m. frame of the collision. The parameters $\Delta=0.581$, $d_{1}=3.979 \mathrm{fm}, d_{2}=0.890 \mathrm{fm}$ are fixed by fitting a two-Gaussian wave function to the Hulthen wave function that well describes the ground state of the deuteron [5]. The process dependence of the nucleon spread can be taken into account using Eqs. (8)-(12). In particular, the longitudinal and transverse spreads in $e^{+} e^{-}$and $p p$ collisions can be set equal and is given by $\sigma \equiv \sigma_{\left(e^{+} e^{-}\right)}=$ $\sigma_{(p p)} / \sqrt{2} \simeq(1.0 \pm 0.1) \mathrm{fm}$.

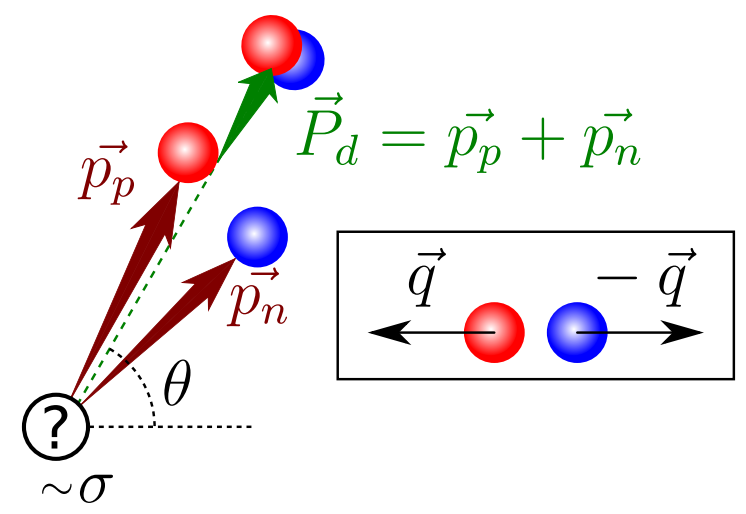

Figure 4: Sketch of the coalescence process in the c.m. frame of the particle collision. A proton with momentum $\mathbf{p}_{p}$ and neutron with momentum $\mathbf{p}_{n}$ may coalesce into a deuteron with momentum $\mathbf{P}_{d}=\mathbf{p}_{p}+\mathbf{p}_{n}$ if their momentum difference in the deuteron rest frame, $2|\mathbf{q}|$, is small. The exact probability is determined by Eq. (13).

The model for helium-3 and tritium production is similar to that for deuteron. In this case, the probability that a proton-proton-neutron or proton-neutron-neutron triplet coalesce is

$$
w=\frac{64}{12} \zeta^{\mathrm{He}} \mathrm{e}^{-b^{2} P^{2}},
$$

with

$$
P^{2}=\frac{1}{3}\left[\left(\mathbf{p}_{1}-\mathbf{p}_{2}\right)^{2}+\left(\mathbf{p}_{1}-\mathbf{p}_{3}\right)^{2}+\left(\mathbf{p}_{2}-\mathbf{p}_{3}\right)^{2}\right]
$$

and

$$
\zeta^{\mathrm{He}}=\frac{b^{2}}{b^{2}+2 \tilde{\sigma}_{\perp}^{2}} \frac{b^{2}}{b^{2}+2 \sigma_{\perp}^{2}} \frac{b^{2}}{b^{2}+2 \sigma_{\|}^{2}} .
$$

Here, $\mathbf{p}_{i}(i=1,2,3)$ is the momentum of the nucleons in the rest frame of the three-particle state. Due to the Gaussian suppression in Eq. (15), one can to a good approximation evaluate the momentum difference of the nucleons entering Eq. (16) in the corresponding two-particle rest frames.

In Eq. (15) the nucleus wave function is approximated by a Gaussian with a rms radius $b$. In Refs. [5, 6] it was set equal to the measured helium-3 rms charge radius $b=1.96 \mathrm{fm}$. However, this choice may lead to an artificially low nucleus yield. Since $\sigma$ is already well constrained by antideuteron measurements, $b$ can be degraded to a free parameter e.g. by setting $b=3^{1 / 3} \sigma_{\left(e^{+} e^{-}\right)}$. 


\section{Summary and Outlook}

The WiFunC model is a per-event coalescence model based on the Wigner function representation of the nucleon and nucleus states. This model includes in a semi-classical picture both two-nucleon momentum correlations provided by a Monte Carlo event generator and the size of the nucleon emission volume. Since the emission volume is process dependent, it explains naturally the differences in (anti)nucleus yields observed in $e^{+} e^{-}, p p$ and $p N$ collisions. The value obtained by fits to experimental data, $\sigma=(1.0 \pm 0.1) \mathrm{fm}$, agrees well with its physical interpretation.

The spread $\sigma$ is currently well constrained by antideuteron data in $p p$ collisions at large energies and large $p_{T}$. Thus, experimental studies on antinuclei production in small interacting systems at low energies in the forward direction is highly warranted. This will reduce uncertainties related to antinuclei production in astrophysical processes, as well as test the WiFunC model. Furthermore, two-particle correlation experiments can be used to directly measure $\sigma$ [7] and test the coalescence hypothesis [35, 36].

The WiFunC model can, in principle, describe the coalescence of nucleons in any particle collision as long as the event generator is able to describe the underlying physics. Currently, the prescription of $\sigma$ is expected to describe well coalescence is small interacting systems, such as $e^{+} e^{-}, p p$ and $p N$ collisions. However, the uncertainties in $\sigma$ will in general increase with increasing system size. Therefore, it would be interesting to instead use the space-time descriptions implemented in event generators like Pythia [21, 22, 25] or UrQMD [23, 37] by evaluating Eq. (7) directly.

\section{Acknowledgments}

I would like to thank S. Ostapchenko and M. Kachelrieß for fruitful collaborations on which this review is based and M. Kachelrieß for comments on this text.

\section{References}

[1] P. von Doetinchem et al., Cosmic-ray Antinuclei as Messengers of New Physics: Status and Outlook for the New Decade, JCAP 08 (2020) 035 [2002 . 04163].

[2] N. Saffold et al., Cosmic Antihelium Nuclei Sensitivity of the GAPS Experiment, 2012.05834

[3] AMS 02 collaboration, The antimatter spectrometer (ams-02): A particle physics detector in space, Nucl. Instrum. Meth. 588 (2008) 227.

[4] H. Caines, The Search for Critical Behavior and Other Features of the QCD Phase Diagram - Current Status and Future Prospects, Nucl. Phys. A967 (2017) 121.

[5] M. Kachelrieß, S. Ostapchenko and J. Tjemsland, Alternative coalescence model for deuteron, tritium, helium-3 and their antinuclei, Eur. Phys. J. A56 (2020) 4 [1905. 01192].

[6] M. Kachelrieß, S. Ostapchenko and J. Tjemsland, Revisiting cosmic ray antinuclei fluxes with a new coalescence model, JCAP 08 (2020) 048 [2002 . 10481]. 
[7] M. Kachelriess, S. Ostapchenko and J. Tjemsland, On nuclear coalescence in small interacting systems, 2012.04352.

[8] A. Schwarzschild and C. Zupancic, Production of Tritons, Deuterons, Nucleons, and Mesons by 30-GeV Protons on A-1, Be, and Fe Targets, Phys. Rev. 129 (1963) 854.

[9] S. T. Butler and C. A. Pearson, Deuterons from high-energy proton bombardment of matter, Phys. Rev. 129 (1963) 836.

[10] P. Chardonnet, J. Orloff and P. Salati, The production of antimatter in our galaxy, Phys. Lett. B409 (1997) 313 [astro-ph/9705110].

[11] M. Kadastik, M. Raidal and A. Strumia, Enhanced anti-deuteron dark matter signal and the implications of PAMELA, Phys. Lett. B683 (2010) 248 [0908 . 1578].

[12] L. A. Dal, Antideuterons as signature for dark matter, master's thesis, NTNU Trondheim, available at http://hdl. handle.net/11250/246403, 2011.

[13] A. Ibarra and S. Wild, Prospects of antideuteron detection from dark matter annihilations or decays at AMS-02 and GAPS, JCAP 1302 (2013) 021 [1209. 5539].

[14] M. W. Winkler and T. Linden, Dark Matter Annihilation Can Produce a Detectable Antihelium Flux through $\bar{\Lambda}_{b}$ Decays, 2006.16251.

[15] L. Csernai and J. I. Kapusta, Entropy and Cluster Production in Nuclear Collisions, Phys. Rept. 131 (1986) 223.

[16] J. Nagle, B. Kumar, D. Kusnezov, H. Sorge and R. Mattiello, Coalescence of deuterons in relativistic heavy ion collisions, Phys. Rev. C 53 (1996) 367.

[17] D.-M. Gomez-Coral, A. Menchaca Rocha, V. Grabski, A. Datta, P. von Doetinchem and A. Shukla, Deuteron and Antideuteron Production Simulation in Cosmic-Ray Interactions, Phys. Rev. D98 (2018) 023012 [1806.09303].

[18] V. Abramov et al., High $p_{T}$ Deuteron and Anti-deuteron Production in pp and $p$ a Collisions at 70-\{GeV\}, Sov. J. Nucl. Phys. 45 (1987) 845.

[19] Y. L. Dokshitzer, V. A. Khoze, A. H. Mueller and S. Troian, Basics of perturbative QCD. 1991.

[20] R. Scheibl and U. W. Heinz, Coalescence and flow in ultrarelativistic heavy ion collisions, Phys. Rev. C59 (1999) 1585 [nucl-th/9809092].

[21] T. Sjöstrand, S. Ask, J. R. Christiansen, R. Corke, N. Desai, P. Ilten et al., An Introduction to PYTHIA 8.2, Comput. Phys. Commun. 191 (2015) 159 [1410.3012].

[22] S. Ferreres-Solé and T. Sjöstrand, The space-time structure of hadronization in the Lund model, Eur. Phys. J. C 78 (2018) 983 [1808. 04619]. 
[23] M. Bleicher et al., Relativistic hadron hadron collisions in the ultrarelativistic quantum molecular dynamics model, J. Phys. G 25 (1999) 1859 [hep-ph/9909407].

[24] T. W. Donnelly, J. A. Formaggio, B. R. Holstein, R. G. Milner and B. Surrow, Foundations of Nuclear and Particle Physics. Cambridge University Press, 2017.

[25] T. Sjöstrand, S. Mrenna and P. Z. Skands, PYTHIA 6.4 Physics and Manual, JHEP 05 (2006) 026 [hep-ph/0603175].

[26] S. Ostapchenko, Monte Carlo treatment of hadronic interactions in enhanced Pomeron scheme: I. QGSJET-II model, Phys. Rev. D83 (2011) 014018 [1010. 1869].

[27] S. Ostapchenko, QGSJET-II: physics, recent improvements, and results for air showers, EPJ Web Conf. 52 (2013) 02001.

[28] ALICE collaboration, Production of deuterons, tritons, ${ }^{3}$ He nuclei and their antinuclei in pp collisions at $\sqrt{s}=$ 0.9, 2.76 and 7 TeV, Phys. Rev. C97 (2018) 024615 [1709. 08522].

[29] ALICE collaboration, Search for a common baryon source in high-multiplicity pp collisions at the LHC, Phys. Lett. B 811 (2020) 135849 [2004.08018].

[30] A. Andronic, P. Braun-Munzinger, K. Redlich and J. Stachel, Decoding the phase structure of QCD via particle production at high energy, Nature 561 (2018) 321 [1710.09425].

[31] J. L. Nagle and W. A. Zajc, Small System Collectivity in Relativistic Hadronic and Nuclear Collisions, Ann. Rev. Nucl. Part. Sci. 68 (2018) 211 [1801.03477].

[32] F. Bellini and A. P. Kalweit, Testing production scenarios for (anti-)(hyper-)nuclei and exotica at energies available at the CERN Large Hadron Collider, Phys. Rev. C 99 (2019) 054905 [1807.05894].

[33] J. Cleymans, S. Kabana, I. Kraus, H. Oeschler, K. Redlich and N. Sharma, Antimatter production in proton-proton and heavy-ion collisions at ultrarelativistic energies, Phys. Rev. C 84 (2011) 054916 [1105 . 3719].

[34] ALICE collaboration, (Anti-)deuteron production in pp collisions at $\sqrt{s}=13 \mathrm{TeV}$, Eur. Phys. J. C 80 (2020) 889 [2003.03184].

[35] F. Bellini, K. Blum, A. P. Kalweit and M. Puccio, On coalescence as the origin of nuclei in hadronic collisions, 2007.01750.

[36] K. Blum and M. Takimoto, Nuclear coalescence from correlation functions, Phys. Rev. C 99 (2019) 044913 [1901.07088].

[37] S. Sombun, K. Tomuang, A. Limphirat, P. Hillmann, C. Herold, J. Steinheimer et al., Deuteron production from phase-space coalescence in the UrQMD approach, Phys. Rev. C 99 (2019) 014901 [1805 . 11509]. 